\title{
CLASSIFICATION THEOREMS FOR $p$-GROUPS AND MODULES OVER A DISCRETE VALUATION RING
}

\author{
BY R. B. WARFIELD, JR. ${ }^{1}$ \\ Communicated by Nathan Jacobson, April 12, 1971
}

In 1933, Ulm gave a complete classification of countable, Abelian $p$-groups in terms of a family of additive, numerical invariants. Recently, R. Nunke discovered a large class of $p$-groups, the totally projective groups, which is a natural extension of the class of countable groups. Ulm's theorem was generalized to this class by P. Hill. The same class was discovered independently, from the point of view of generators and relations, by Crawley and Hales. In this note we announce three generalizations of this theory. We introduce two new classes of mixed modules over a discrete valuation ring, and a new class of $p$-groups (containing the totally projective groups). In each case, we also introduce a new family of invariants which can be used, together with Ulm's invariants, to prove a classification theorem.

In the following, $R$ will be a discrete valuation ring, $R$ will be the ring regarded as a module over itself, and $p$ will be a generator of the maximal ideal of $\boldsymbol{R}$. If $M$ is any $\boldsymbol{R}$-module, we define $p^{\alpha} M$ for any ordinal $\alpha$ by $p^{\alpha+1} M=p\left(p^{\alpha} M\right)$ and, if $\alpha$ is a limit ordinal, $p^{\alpha} M=\bigcap_{\beta<\alpha} p^{\beta} M$. We recall that a module $M$ is reduced if it has no nonzero divisible submodules, or, equivalently, if for some ordinal $\alpha, p^{\alpha} M=0$. A module is a $T$-module [1] if it can be given in terms of generators and relations in such a way that all of the relations have the form $p x=y$ or $p x=0$. A torsion module $M$ is totally projective [7] if for any module $N$ and any ordinal $\alpha, p^{\alpha} \operatorname{Ext}\left(M / p^{\alpha} M, N\right)$ $=0$. The work of Nunke [7], Crawley and Hales [1], and Hill [2], suitably generalized to modules over a discrete valuation ring, shows that a torsion, reduced module is a $T$-module if and only if it is totally projective, and that these modules can be classified in terms of their Ulm invariants (defined below).

We will call a class $\mathscr{C}$ of $R$-modules closed if it satisfies the following conditions:

(i) A module isomorphic to a module in $\mathscr{C}$ is in $\mathscr{C}$.

(ii) A direct sum of modules in $\mathscr{C}$ is in $\mathscr{C}$.

AMS 1970 subject classifications. Primary 13C05, 20K99; Secondary 18G25, 20K40.

Key words and phrases. Abelian groups, $p$-groups, modules over a discrete valuation ring, Ulm's theorem, pure submodules, high subgroups, invariants for modules.

${ }^{1}$ Supported by GP-28946. 
(iii) A summand of a module in $\mathscr{C}$ is in $\mathscr{C}$.

(iv) If $M$ is a module and $\alpha$ an ordinal, then $M$ is in $\mathscr{C}$ if and only if $p^{\alpha} M$ and $M / p^{\alpha} M$ are in $\mathscr{C}$.

1. Ulm's theorem for mixed modules. If

$$
0 \rightarrow A \rightarrow B \rightarrow C \rightarrow 0
$$

is a short exact sequence of $R$-modules, we say that (1) is $h$-pure if for every ordinal $\alpha$, the sequence

$$
0 \rightarrow p^{\alpha} A \rightarrow p^{\alpha} B \rightarrow p^{\alpha} C \rightarrow 0
$$

is exact. A module $P$ is $h$-pure-projective if for every $h$-pure short exact sequence (1), the natural map $\operatorname{Hom}(P, B) \rightarrow \operatorname{Hom}(P, C)$ is surjective.

If $\lambda$ is a limit ordinal, we say that $M$ is a $\lambda$-elementary $K T$-module if $p^{\lambda} M \cong R$ and $M / p^{\lambda} M$ is a reduced torsion $T$-module.

THEOREM 1. A reduced $R$-module $M$ is $h$-pure-projective if and only if it is the direct sum of a torsion T-module and a family of $\lambda$-elementary $K T$ modules (for various $\lambda$ ).

We call such a module a $K T$-module.

THEOREM 2. The class of KT-modules is closed, and if $\mathscr{C}$ is any closed class of modules containing $R$, then $\mathscr{C}$ contains the KT-modules.

There is a natural way to extend Ulm's invariants to mixed modules in such a way that one gets all of the original invariants together with a new family of invariants indexed by limit ordinals. This was first pointed out by Kulikov [5], who proved a theorem equivalent to our Theorem 3 below for countably generated modules.

For any module $M$, we denote by $M[p]$ the submodule generated by elements of order $p$. We define

$$
U_{\alpha}(M)=\left(p^{\alpha} M\right)[p] /\left(p^{\alpha+1} M\right)[p] \quad(\alpha \geqq 0)
$$

and let $f(\alpha, M)=\operatorname{dim}\left(U_{\alpha}(M)\right)$, where the dimension is taken over the field $\boldsymbol{R} / p \boldsymbol{R}$. The cardinals $f(\alpha, M)$ are the Ulm invariants of $M$. For any $\boldsymbol{R}$-module $M$, and limit ordinal $\lambda$, we define

$$
V_{\lambda}(M)=p^{\lambda} M /\left(p^{\lambda+1} M+T_{\lambda}\right)
$$

where $T_{\lambda}$ is the torsion submodule of $p^{\lambda} M$, and we let $h(\lambda, M)=\operatorname{dim}\left(V_{\lambda}(M)\right)$.

We remark that if $M$ is a $K T$-module and $\lambda$ is a limit ordinal, then $p^{\lambda} M / p^{\lambda+\omega} M$ is a direct sum of cyclic modules. The number $h(\lambda, M)$ is the number of summands isomorphic to $R$ in a decomposition of $p^{\lambda} M / p^{\lambda+\omega} M$, 
while the number $f(\lambda+n, M)$ is the number of summands isomorphic to $R / p^{n+1} R$.

THEOREM 3. If $A$ and $B$ are $K T$-modules, then $A \cong B$ if and only if for all ordinals $\alpha$ and all limit ordinals $\lambda, f(\alpha, A)=f(\alpha, B)$ and $h(\lambda, A)=h(\lambda, B)$. $A$ $K T$-module $A$ is torsion if and only if $h(\lambda, A)=0$ for all limit ordinals $\lambda$.

THEOREM 4. Let $\mathscr{C}$ be a class of modules containing the KT-modules and such that the direct sum of two modules in $\mathscr{C}$ is in $\mathscr{C}$. Suppose that $\mathscr{C}$ has the property that two modules $A$ and $B$ in $\mathscr{C}$ are isomorphic if for every ordinal $\alpha$ and every limit ordinal $\lambda, f(\alpha, A)=f(\alpha, B)$ and $h(\lambda, A)=h(\lambda, B)$. Then $\mathscr{C}$ coincides with the class of KT-modules.

2. A classification theorem for $p$-groups. In this section we are interested only in $p$-groups, which we regard as torsion modules over the ring $Z_{p}$ (the integers localized at $p$ ). An $S$-group is a group which is isomorphic to the torsion submodule of a $K T$-module (over the ring $Z_{p}$ ). We recall that if $H$ is a subgroup of $G$, then $H$ is $\lambda$-high if $H \cap p^{\lambda} G=0$ and $H$ is maximal with respect to this property.

THEOREM 5. The class of S-groups is a closed class with the additional property that, for any limit ordinal $\lambda$, a $\lambda$-high subgroup of an $S$-group is an $S$-group. If $\mathscr{C}$ is a closed class of p-groups containing $Z / p Z$ and with the property that for any limit ordinal $\lambda$, a $\lambda$-high subgroup of a group in $\mathscr{C}$ is in $\mathscr{C}$, then $\mathscr{C}$ contains the class of $S$-groups.

If $G$ is an $S$-group and is isomorphic to the torsion submodule of the $K T$-module $M$, then it can be shown that if $\lambda$ is a limit ordinal which is not cofinal with $\omega$, then $h(\lambda, M)$ is an invariant of $G$, so we can define $k(\lambda, G)$ $=h(\lambda, M)($ where the restriction that $\lambda$ is a limit ordinal not cofinal with $\omega$ is essential). Using cotorsion envelopes [6], this can be done in a more functorial manner. For any $Z_{p}$-module $M$, we let $C_{\lambda}$ be the cotorsion envelope of $M / p^{\lambda} M$ and let $k(\lambda, M)$ be the $Z / p Z$-dimension of $p^{\lambda} C_{\lambda} / p^{\lambda+1} C_{\lambda}$.

THEOREM 6. If $A$ and $B$ are $S$-groups, then $A \cong B$ if and only if for every ordinal $\alpha$ and every limit ordinal $\lambda$ which is not cofinal with $\omega, f(\alpha, A)$ $=f(\alpha, B)$ and $k(\lambda, A)=k(\lambda, B)$. If $A$ is an $S$-group, then $A$ is a T-group if and only if for every limit ordinal $\lambda$ which is not cofinal with $\omega, k(\lambda, A)=0$.

THEOREM 7. Let $\mathscr{C}$ be a class of p-groups containing the S-groups and such that the direct sum of two groups in $\mathscr{C}$ is in $\mathscr{C}$. Suppose that $\mathscr{C}$ has the property that two groups $A$ and $B$ in $\mathscr{C}$ are isomorphic if for every ordinal $\alpha$ and every limit ordinal $\lambda$ which is not cofinal with $\omega, f(\alpha, A)=f(\alpha, B)$ and $k(\lambda, A)$ $=k(\lambda, B)$. Then $\mathscr{C}$ coincides with the class of $S$-groups.

3. Modules defined by generators and relations. In this section we outline a generalization of the results of $\S 1$ for mixed modules over a discrete 
valuation ring $\boldsymbol{R}$. We define the height $h(x)$ of an element in an $R$-module $M$ by setting $h(x)=\alpha$ if $x \in p^{\alpha} M$ and $x \notin p^{\alpha+1} M$, and $h(x)=\infty$ if $x \in p^{\alpha} M$ for all ordinals $\alpha$. An Ulm sequence is a sequence $\left\{\alpha_{i}\right\}, i \geqq 0$, such that each entry is an ordinal or $\infty$ and such that (i) if $\alpha_{i}=\infty$ then $\alpha_{i+1}=\infty$ and (ii) if $\alpha_{i+1} \neq \infty$ then $\alpha_{i+1}>\alpha_{i}$. If $M$ is a module and $x \in M$, the Ulm sequence of $x, U(x)$, is the sequence $\left\{\alpha_{i}\right\}$ given by $\alpha_{i}=h\left(p^{i} x\right)$. If $s=\left\{\alpha_{i}\right\}$ and $u=\left\{\beta_{i}\right\}$ are two Ulm sequences, we say $s \geqq u$ if $\alpha_{i} \geqq \beta_{i}$ for all $i \geqq 0$. If $M$ is an $R$-module and $u$ an Ulm sequence, we can define a fully invariant submodule of $M$ by defining

$$
u M=\{x \in M: U(x) \geqq u\} .
$$

It was shown by Kaplansky [3] that if $M$ is a countably generated torsion module then all fully invariant submodules of $M$ are of this form.

We say a short exact sequence (1) of $R$-modules is $s$-pure if for any Ulm sequence $u$, the sequence

$$
0 \rightarrow u A \rightarrow u B \rightarrow u C \rightarrow 0
$$

is exact. A module if $s$-pure-projective if for every $s$-pure short exact sequence (1), the natural map $\operatorname{Hom}(P, B) \rightarrow \operatorname{Hom}(P, C)$ is surjective.

We recall that a $T$-module is a module given by generators and relations in such a way that all of the relations are of the form $p x=y$ or $p x=0$.

THEOREM 8. A module is s-pure-projective if and only if it is a summand of a T-module.

A summand of a $T$-module is called a $T^{*}$-module. We remark that $K T$-modules and divisible modules are $T$-modules. An examination of the generators and relations enables one to conclude easily that any T-module is a direct sum of modules of torsion-free rank at most one. If $M$ is a module of torsion-free rank one and $x$ and $y$ are two elements of infinite order, with $U(x)=\left\{\alpha_{i}\right\}$ and $U(y)=\left\{\beta_{i}\right\}$, then $U(x)$ and $U(y)$ are equivalent [4] in the sense that there are integers $m$ and $n$ such that $\alpha_{n+i}=\beta_{m+i}$ for all $i \geqq 0$. This equivalence class of Ulm sequences is denoted $U(M)$ and is an invariant of $M$.

If $M$ is an $R$-module which is a direct sum of modules of torsion-free rank one, $M=\bigoplus_{i \in I} M_{i}$, and $e$ is an equivalence class of Ulm sequences, we would like to define $g(e, M)$ to be the number of summands $M_{i}$ with $U\left(M_{i}\right)=e$. To show that $g(e, M)$ is an invariant of $M$ we must look at a new category $\mathscr{H}$. The objects of $\mathscr{H}$ are the $R$-modules, and the homomorphism group $\operatorname{Hom}_{\mathscr{H}}(M, N)$ is defined in terms of equivalence classes of ordinary homomorphisms defined on submodules of $M$. The elements of the equivalence classes are homomorphisms $f: A \rightarrow N$ where $A$ is a torsion-free submodule of $M$ such that $M / A$ is torsion, and where $f$ 
satisfies the restriction $h(f(a)) \geqq h(a)$, for all $a \in A$, and where $h(a)$ is computed in $M$ (so that the restriction is nontrivial). If $g: B \rightarrow N$ is another such homomorphism, then $f$ and $g$ are equivalent if they agree on $A \cap B$.

A module of torsion-free rank one is indecomposable in $\mathscr{H}$, and two modules $M$ and $N$ of torsion-free rank one are isomorphic in $\mathscr{H}$ if and only if $U(M)=U(N)$. A suitable generalization of the Krull-Schmidt theorem proved in [8] enables one to conclude that if $M$ is a module which is a direct sum of modules of torsion-free rank one, then $g(e, M)$ is an invariant of $M$, and that any summand of $M$ is isomorphic in $\mathscr{H}$ to a direct sum of modules of torsion-free rank one. In particular, the invariants $g(e, M)$ are defined for all $T^{*}$-modules.

THEOREM 9. If $M$ and $N$ are reduced $T^{*}$-modules, then $M \cong N$ if and only if for every ordinal $\alpha$ and every equivalence class $e$ of Ulm sequences, $f(\alpha, M)=f(\alpha, N)$ and $g(e, M)=g(e, N)$.

\section{REFERENCES}

1. P. Crawley and A. W. Hales, The structure of abelian p-groups given by certain presentations, J. Algebra 12 (1969), 10-23. MR 39 \#307.

2. P. Hill, On the classification of abelian groups (to appear).

3. I. Kaplansky, Infinite abelian groups, rev. ed., Univ. of Michigan Press, Ann Arbor, Mich., 1969. MR 38 \#2208.

4. I. Kaplansky and G. W. Mackey, A generalization of Ulm's theorem, Summa Brasil. Math. 2 (1951), 195-202. MR 14, 128.

5. L. Kulikov, Generalized primary groups. I, II, Trudy Moskov Mat. Obšč. 1 (1952), 247-326; ibid. 2 (1953), 85-167. (Russian) MR 14, 132; MR 15, 9.

6. E. Matlis, Cotorsion modules, Mem. Amer. Math. Soc. No. 49 (1964). MR 31 \# 2283.

7. R. J. Nunke, Homology and direct sums of countable abelian groups, Math. Z. 101 (1967), 182-212. MR 36 \# 1538.

8. R. B. Warfield, Jr., A Krull-Schmidt theorem for infinite sums of modules, Proc. Amer. Math. Soc. 22 (1969), 460-465. MR 39 \#4213.

Department of Mathematics, University of Washington, Seattle, Washington 98105 